\title{
Investigation of transit-selected exoplanet candidates from the MACHO survey ${ }^{\star}$
}

\author{
S. D. Hügelmeyer ${ }^{1}$, S. Dreizler ${ }^{1}$, D. Homeier ${ }^{1}$, and A. Reiners ${ }^{1,2}$ \\ 1 Institut für Astrophysik, Georg-August-Universität Göttingen, Friedrich-Hund-Platz 1, 37077 Göttingen, Germany \\ ${ }^{2}$ Hamburger Sternwarte, Universität Hamburg, Gojenbergsweg 112, 21029 Hamburg, Germany
}

Received 22 January 2007 / Accepted 22 March 2007

\section{ABSTRACT}

\begin{abstract}
Context. Planets outside our solar system transiting their host star, i.e. those with an orbital inclination near $90^{\circ}$, are of special interest to derive physical properties of extrasolar planets. With the knowledge of the host star's physical parameters, the planetary radius can be determined. Combined with spectroscopic observations the mass and therefore the density can be derived from Dopplermeasurements. Depending on the brightness of the host star, additional information, e.g. about the spin-orbit alignment between the host star and planetary orbit, can be obtained.

Aims. The last few years have witnessed a growing success of transit surveys. Among other surveys, the MACHO project provided nine potential transiting planets, several of them with relatively bright parent stars. The photometric signature of a transit event is, however, insufficient to confirm the planetary nature of the faint companion. The aim of this paper therefore is a determination of the spectroscopic parameters of the host stars as well as a dynamical mass determination through Doppler-measurements.

Methods. We obtained follow-up high-resolution spectra for five stars selected from the MACHO sample, which are consistent with transits of low-luminosity objects. Radial velocities were determined by means of cross-correlation with model spectra. The MACHO light-curves were compared to simulations based on the physical parameters of the system derived from the radial velocities and spectral analyses.

Results. We show that all transit light-curves of the exoplanet candidates analysed in this work can be explained by eclipses of stellar objects, hence none of the five transiting objects is a planet.
\end{abstract}

Key words. stars: planetary systems - eclipses - techniques: radial velocities - stars: binaries: eclipsing stars: binaries: spectroscopic

\section{Introduction}

After the first detections of planets outside our solar system (Wolszczan \& Frail 1992; Mayor \& Queloz 1995), an intensive search with various methods began (see Schneider 2002, for an overview); more than 200 planets have been found to date (http://exoplanet.eu/). Most of these exoplanet detections have been performed via the radial velocity $(R V)$ method where the "wobble" of the parent star caused by the planet is measured by spectral line shifts. Since these effects are very small for low-mass planets in orbits of tens to hundreds of days, the determination of orbital period, phase, inclination, eccentricity, and $R V$ amplitude demands $R V$ accuracies of a few meters per second (Marcy et al. 2000).

Meanwhile, alternative methods for planet detections have also been successfully applied. The first four microlensing planets were detected (Bond et al. 2004; Udalski et al. 2005; Beaulieu et al. 2006; Gould et al. 2006), possible first direct images of extra-solar planets were published (Chauvin et al. 2004, 2005a,b; Neuhäuser et al. 2005; Biller et al. 2006), and the number of detections due to transit searches is steadily increasing (McCullough et al. 2006; Bakos et al. 2007; O'Donovan et al. 2006; Collier Cameron et al. 2007).

The transit method is of special interest, since it permits the derivation of additional physical parameters of the planet, e.g. the radius can be measured either indirectly via the radius of

* Based on observations made with ESO Telescopes at the La Silla or Paranal Observatories under programme ID 075.C-0526(A). the host star or directly via detection of the secondary eclipse as observed with the Spitzer Space Telescope (Charbonneau et al. 2005; Deming et al. 2005). If combined with a radial velocity variation measurement, the mass and mean density can be determined, revealing constraints for the planetary structure. Furthermore, transiting systems allow us to investigate the atmospheres of the planets (Charbonneau et al. 2002; Vidal-Madjar et al. 2004) as well as the spin-orbit-alignment between the rotational axis of the host star and the planetary orbit (Wolf et al. 2007; Gaudi \& Winn 2007; Winn et al. 2006).

Drake \& Cook (2004, hereafter DC) published a list of nine restrictively selected, transiting planet candidates from the MACHO project (Alcock et al. 1992). Only transit light-curves with no indication of gravitational distortion as well as clear U-shaped transit events were considered. De-reddened colours as well as light-curve fitting provide a good estimate of the companion radius. Only companions below 3 Jupiter radii were selected.

Based on high-resolution spectra, the orbital velocities of five potential host stars of exoplanet candidates were measured. We analysed the $R V$ measurements together with MACHO transit light-curves in order to determine the system parameters complemented by a spectral analysis.

The paper is organized as follows: in the next section, we briefly describe the spectroscopic observations and the spectral analysis as well as the Doppler-measurements; in Sect. 3, we describe the results of the individual systems and summarise in Sect. 4. 
Table 1. Orbital elements, rotation velocities, and stellar parameters for all five analysed systems. Components $c$ and $d$ of MACHO 118.18272.189 and component $b$ of MACHO 120.22041.3265 are not visible in the spectra. $P_{\text {MACHO }}$ is taken from Drake \& Cook (2004) and $P$ denotes the period derived using the light-curves and $R V$ measurements. $K$ is the semi-amplitude of the $R V$ variations, $V_{0}$ the system velocity, and $i$ the orbital inclination. In cases of systems with elliptical orbits, $e$ is the eccentricity and $\omega$ the longitude of the periastron. Furthermore, the mass $M^{R V}$ is given in cases where the $R V$ amplitude of two components is known. Then $T_{\text {eff }}^{R V}$ and $R$ are calculated for zero- and terminal-age main sequence models. $T_{\text {eff }}^{\mathrm{SA}}$ is the effective temperature derived from the spectral analyses. In cases where just $T_{\mathrm{eff}}^{\mathrm{SA}}$ from the spectral analyses is known, $M^{\mathrm{SA}}$ and $R$ are derived masses and radii from evolution models. All values in this table relate to the assumption of zero-age main sequence stars.

\begin{tabular}{|c|c|c|c|c|c|c|c|c|c|c|c|c|c|}
\hline MACHO ID & & $\begin{array}{c}P \\
\text { [days] }\end{array}$ & $\begin{array}{c}P_{\mathrm{MACHO}} \\
\text { [days] }\end{array}$ & $\begin{array}{c}K \\
{\left[\mathrm{~km} \mathrm{~s}^{-1}\right]}\end{array}$ & $\begin{array}{c}V_{0} \\
{\left[\mathrm{~km} \mathrm{~s}^{-1}\right]}\end{array}$ & $\begin{array}{c}i \\
{\left[{ }^{\circ}\right]}\end{array}$ & $e$ & $\begin{array}{l}\omega \\
{\left[{ }^{\circ}\right]}\end{array}$ & $\begin{array}{l}M^{R V} \\
{\left[M_{\odot}\right]}\end{array}$ & $\begin{array}{l}T_{\text {eff }}^{R V} \\
{[\mathrm{~K}]}\end{array}$ & $\begin{array}{l}T_{\text {eff }}^{\mathrm{SA}} \\
{[\mathrm{K}]}\end{array}$ & $\begin{array}{c}M^{\mathrm{SA}} \\
{\left[M_{\odot}\right]}\end{array}$ & $\begin{array}{c}R \\
{\left[R_{\odot}\right]}\end{array}$ \\
\hline 118.18272 .189 & $\begin{array}{l}a \\
b \\
c \\
d\end{array}$ & $\begin{array}{c}- \\
- \\
3.9346 \\
3.9346\end{array}$ & $\begin{array}{c}1.9673 \\
- \\
- \\
-\end{array}$ & $\begin{array}{c}0.00 \\
0.00 \\
- \\
-\end{array}$ & $\begin{array}{c}-25.51 \pm 0.03 \\
+05.46 \pm 0.03 \\
- \\
-\end{array}$ & $\begin{array}{c}- \\
- \\
(90.0)\end{array}$ & $\begin{array}{l}- \\
- \\
- \\
-\end{array}$ & $\begin{array}{l}- \\
- \\
- \\
-\end{array}$ & $\begin{array}{c}- \\
- \\
0.41 \\
0.41\end{array}$ & $\begin{array}{c}- \\
- \\
3730 \\
3730\end{array}$ & $\begin{array}{c}5800 \\
5800 \\
- \\
-\end{array}$ & $\begin{array}{l}- \\
- \\
- \\
-\end{array}$ & $\begin{array}{c}- \\
- \\
0.38 \\
0.38\end{array}$ \\
\hline 118.18407 .57 & $\begin{array}{l}a \\
b \\
c\end{array}$ & $\begin{array}{c}4.7972 \\
- \\
4.7972\end{array}$ & $\begin{array}{c}2.3986 \\
- \\
2.3986\end{array}$ & $\begin{array}{c}78.84 \pm 0.10 \\
0.00 \\
89.68 \pm 0.09\end{array}$ & $\begin{array}{l}-20.48 \pm 0.07 \\
-08.39 \pm 0.03 \\
-20.48 \pm 0.06\end{array}$ & 84.0 & $\begin{array}{l}- \\
- \\
-\end{array}$ & $\begin{array}{l}- \\
- \\
-\end{array}$ & $\begin{array}{c}1.27 \\
- \\
1.11\end{array}$ & $\begin{array}{c}6430 \\
- \\
5980\end{array}$ & $\begin{array}{l}6200 \\
6600 \\
6200\end{array}$ & $\begin{array}{l}- \\
- \\
-\end{array}$ & $\begin{array}{c}1.23 \\
- \\
1.04\end{array}$ \\
\hline 118.18793 .469 & $\begin{array}{l}a \\
b\end{array}$ & $\begin{array}{l}4.0744 \\
4.0744\end{array}$ & $\begin{array}{l}2.0372 \\
2.0372\end{array}$ & $\begin{array}{l}75.81 \pm 0.18 \\
83.67 \pm 0.25\end{array}$ & $\begin{array}{l}-56.30 \pm 0.11 \\
-56.30 \pm 0.14\end{array}$ & 85.6 & 0.041 & 89.94 & $\begin{array}{l}0.90 \\
0.82\end{array}$ & $\begin{array}{l}5140 \\
5070\end{array}$ & $\begin{array}{l}5400 \\
5400\end{array}$ & - & $\begin{array}{l}0.81 \\
0.76\end{array}$ \\
\hline 120.22041 .3265 & $\begin{array}{l}a \\
b\end{array}$ & $\begin{array}{l}5.4083 \\
5.4083\end{array}$ & $\begin{array}{l}5.4083 \\
5.4083\end{array}$ & $\begin{array}{c}22.18 \pm 0.06 \\
114.90\end{array}$ & $\begin{array}{l}-24.00 \pm 0.04 \\
-24.00 \pm 0.04\end{array}$ & 89.8 & 0.108 & 19.98 & - & $\begin{array}{l}- \\
-\end{array}$ & $\begin{array}{c}6200 \\
(3340)\end{array}$ & $\begin{array}{c}1.19 \\
(0.23)\end{array}$ & $\begin{array}{l}1.15 \\
0.28\end{array}$ \\
\hline 402.47800 .723 & $\begin{array}{l}a \\
b \\
c\end{array}$ & $\begin{array}{c}8.5496 \\
- \\
8.5496\end{array}$ & $\begin{array}{c}4.2748 \\
- \\
4.2748\end{array}$ & $\begin{array}{c}75.91 \pm 0.04 \\
0.00 \\
68.09 \pm 0.07\end{array}$ & $\begin{array}{l}+00.40 \pm 0.04 \\
-26.40 \pm 0.04 \\
+00.40 \pm 0.07\end{array}$ & 85.8 & $\begin{array}{l}- \\
- \\
-\end{array}$ & $\begin{array}{l}- \\
- \\
-\end{array}$ & $\begin{array}{c}1.26 \\
- \\
1.40\end{array}$ & $\begin{array}{c}6400 \\
- \\
6820\end{array}$ & $\begin{array}{l}6400 \\
5800 \\
6400\end{array}$ & $\begin{array}{l}- \\
- \\
-\end{array}$ & $\begin{array}{c}1.22 \\
- \\
1.37\end{array}$ \\
\hline
\end{tabular}

\section{Observations and analyses}

In period 75 we secured three spectra for each of the five brightest candidates. We used ESO's Fibre-fed Extended Range Échelle Spectrograph (FEROS) mounted on the $2.2 \mathrm{~m}$ telescope at La Silla, Chile. The spectrograph provides a spectral resolution of $R \sim 48000$ and covers a wavelength range from $3500 \AA$ to $9200 \AA$. The instrumental specifications list a rms velocity error of $\sim 25 \mathrm{~m} \mathrm{~s}^{-1}$. This is sufficient to detect faint low-mass star companions and distinguish them from sub-stellar companions, which was the primary aim of the observations. The secondary aim is to use the spectra for a spectral analysis in order to derive the stellar parameters of the host stars.

The observations of the five targets have been performed between August 19 and September 16, 2005. For each object we have obtained three spectra with exposure times between $2400 \mathrm{~s}$ and $3500 \mathrm{~s}$, depending on the brightness of the object. The signal-to-noise ratio is $\sim 10$.

The data were reduced using the FEROS Data Reduction System (DRS). The échelle spectra were corrected for bias and flat field and wavelength was calibrated. The calibration was additionally quality checked by cross-correlating the observation with a sky line spectrum. The spectra were then corrected by applying relative wavelength shifts. Barycentric and Earth rotation velocity influences on the wavelengths are accounted for automatically by the DRS.

For the determination of the radial velocities we used the extracted FEROS and synthetic spectra of main sequence model stars calculated from LTE model atmospheres using PHOENIX (Hauschildt et al. 1999) version 14.2. Both spectra were normalised and relative fluxes were interpolated on a logarithmic wavelength scale. A cross-correlation (CC) between a model with $T_{\text {eff }}=5600 \mathrm{~K}$ and observation was performed between $5000 \AA$ and $7000 \AA$. The CC was implemented using a grid with 200 steps of $\Delta \log (\lambda /[\AA])=2.2 \times 10^{-6}$ in each direction. This method turned out to be robust against the use of different model spectra. We could identify up to three spectroscopic components in our data. Each of the peaks in the CC was then fitted by a Gaussian and the position of the maximum of the fit gives the radial velocity. The errors of the $R V$ measurements were calculated from the standard deviation of the Gaussian plus the accuracy limit of FEROS of $25 \mathrm{~m} \mathrm{~s}^{-1}$. These $R V$ errors are in the range between 50 and $350 \mathrm{~m} \mathrm{~s}^{-1}$.

The $\mathrm{CC}$ function was also used to determine the projected rotation velocities of the stars. We therefore applied a solar spectrum as template convolved with rotational profiles following Gray (2005). This method allows the derivation of stellar radii in binaries, assuming a synchronised orbit. In this analysis, the determined rotational velocity $v \sin i$ is of the order of the uncertainty in most cases, which, due to the low signal-to-noise ratio, is about $5 \mathrm{~km} \mathrm{~s}^{-1}$. These derived radii are consistent with the ones obtained from main sequence models (see Table 1). Additional constraints for the radii of the binary components visible in the spectra can therefore not be derived.

In order to spectroscopically identify the components of the analysed systems, we again used the PHOENIX model grid which ranges from $4000 \mathrm{~K}$ to $6800 \mathrm{~K}$ in $T_{\text {eff }}$ and from -1.5 to 0.0 in relative solar metallicity $[\mathrm{Fe} / \mathrm{H}]$. It should be noted that this is not sufficient for a detailed abundance determination, which was not the aim of this work. The surface gravity is kept constant at $\log g=4.5$. Knowing the $R V$ of the individual components of a system, the models were gauss-folded to the resolution of the observation and shifted to their position in the observed spectrum. Depending on the number of spectral components, all possible combinations of model spectra were tested for each observed spectrum. A $\chi^{2}$-test was used to identify the best fitting models. Given the low signal-to-noise ratio of the spectra, we estimate an uncertainty of about $400 \mathrm{~K}$ in effective temperature.

In cases where we know the $R V$ amplitudes for two components (MACHO 118.1407.57, 118.18793.469, 402.47800.723), $M \sin i$ is known for both components. Assuming $i=90^{\circ}$ for the first iteration, we determined radii and effective temperatures $\left(T_{\text {eff }}^{R V}\right.$ in Table 1) from interpolation of the Geneva model tracks (Schaerer et al. 1993) assuming zero-age main sequence 
(ZAMS) or terminal-age main sequence (TAMS) stars. We then applied the eclipsing binary simulation software Nightfall ${ }^{1}$ with the derived stellar and orbital parameters from the previous step as input and calculated a best model fit to the $R$-band lightcurve and radial velocity measurements simultaneously. We used the third light contribution and the inclination as free parameters and calculated $\chi^{2}$-values for the light-curve fits assuming ZAMS and TAMS stars. In the second iteration, we repeated the fit with the now known inclination (see Fig. 3). For these three systems the so-derived effective temperature can be compared to the one of the spectral analyses $\left(T_{\mathrm{eff}}^{\mathrm{SA}}\right.$ in Table 1$)$. Deviations are within our estimated uncertainties and show the overall consistency of our main-sequence solution.

In the other two cases (MACHO 120.22041.3265 and MACHO 118.18272.189), the effective temperature from the spectral analysis was used to derive masses and radii of each component, again assuming ZAMS and TAMS stars. In the lightcurve simulations for the MACHO $R$-band photometry we varied the inclination and the radius $R_{2}$ of the potential transiting object, assuming ZAMS and TAMS primary stars.

\section{Results}

We will present results for the five targeted MACHO objects for which we found an orbital solution that explains the detected transits and the measured radial velocities. In Fig. 1 we show fitted light-curves to the photometric MACHO data (bottom panels in the plots) and $R V$-curve fits to the Doppler-measurements (asterisks in the top panel of the plots). The dashed lines are for circular orbits and the solid lines show a best-fit elliptical orbit. Figure 2 again shows Nightfall light-curve solutions to the photometric data as well as the $R V$ fits to the Dopplermeasurements. Here circular orbits reproduce the observations best. All stars were assumed to be on the ZAMS for the fits in Figs. 1 and 2. $\chi^{2}$-contour plots for both ZAMS and TAMS stars are depicted in Fig. 3. The inclination of the orbital plane and the third light contribution (bottom three plots) and the radius $R_{2}$ (top two plots) of the potential transiting objects were treated to vary. A list of the orbital parameters $P_{\mathrm{MACHO}}$ (period given by Drake \& Cook 2004), the derived period $P$ in our analyses, the $R V$ amplitude $K$, the system velocity $V_{0}$, the orbital inclination $i$, and in cases of systems with elliptical orbits, $e$ the eccentricity and $\omega$ the longitude of the periastron as well as the mass, effective temperature, and radius is shown in Table 1.

\section{MACHO 120.22041.3265}

MACHO 120.22041 .3265 is the only system in our sample with just one component visible in the spectra. Spectral analysis yields $T_{\mathrm{eff}}=6200 \mathrm{~K}$ and indicates a low metallicity $([\mathrm{Fe} / \mathrm{H}]=-1.0)$. The fit of a sinusoidal to the $R V \mathrm{~s}$ folded to a period of $5.4083 \mathrm{~d}$ (DC, dashed curve in Fig. 1) differs from the $R V$ measurement at a phase of 0.87 by $\sim 10 \mathrm{~km} \mathrm{~s}^{-1}$. A better fit is provided by an elliptical orbit with an eccentricity of $e=0.108$, a longitude of periastron of $\omega=19.98$, and an orbital semiamplitude of $K=22.18 \mathrm{~km} \mathrm{~s}^{-1}$. For such a system the radius and mass of the secondary is $R_{2}=0.3 R_{\odot}$ and $M_{2}=0.23 M_{\odot}$ for a ZAMS and $R_{2}=0.5 R_{\odot}$ and $M_{2}=0.25 M_{\odot}$ for a TAMS primary (see Fig. 3), clearly indicating an M-dwarf companion. With these parameters, the system is very similar to OGLE-TR78 (Pont et al. 2005).

${ }^{1}$ http://www.hs.uni-hamburg.de/DE/Ins/Per/Wichmann/ Nightfall.html

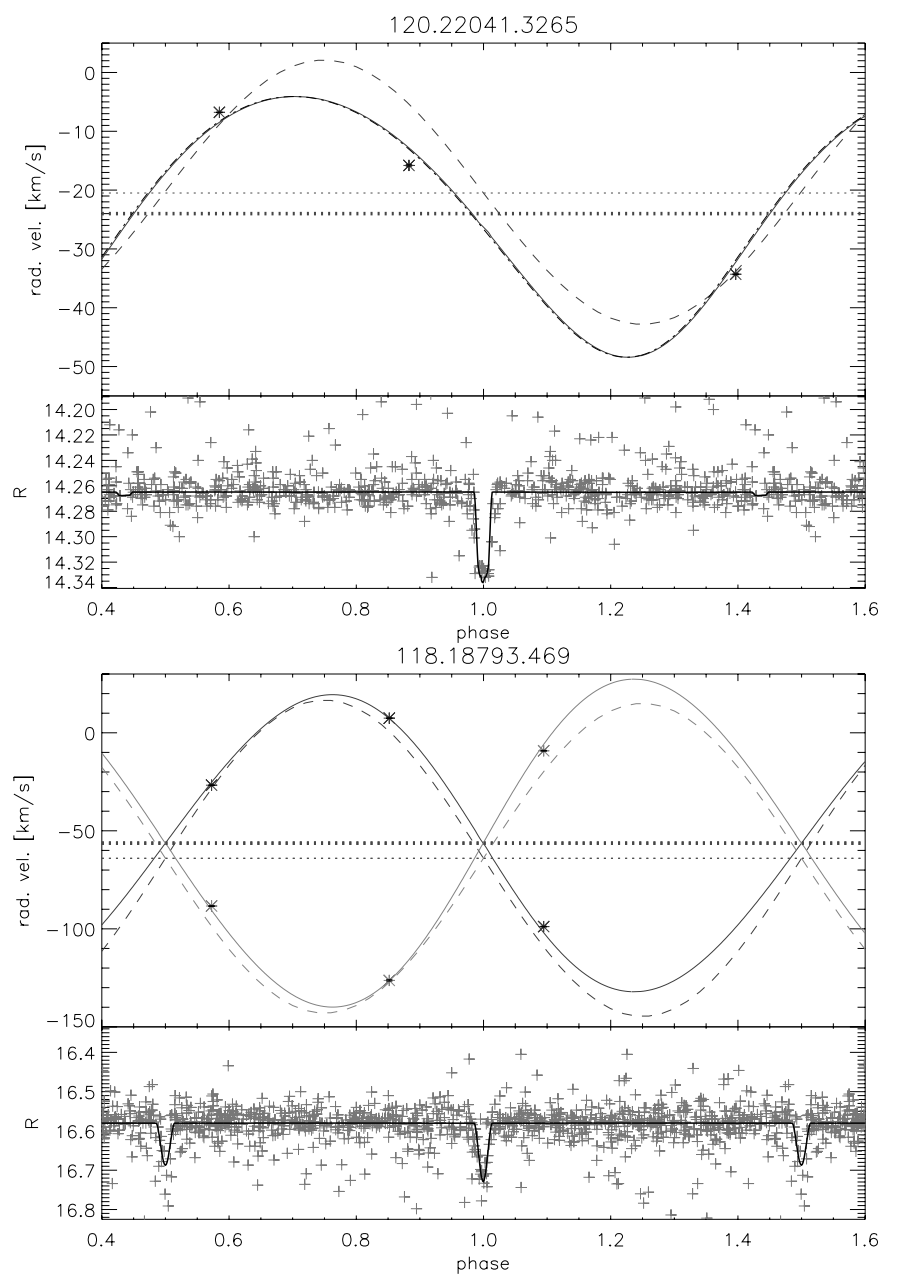

Fig. 1. Radial-velocity and light-curve fits for systems with elliptical orbits. The dashed lines show best-fit sinusoidals while the solid lines show best-fit eccentric orbits. Component $a$ is plotted in black, component $b$ in grey. The system velocity for the circular orbit is shown by the thin, and for the elliptical orbit by the thick dotted line. The solutions shown are calculated assuming ZAMS stars. The error bars for the $R V$ measurements are of the size of the symbols.

We used Eq. (6.2) of Zahn (1977) to calculate an estimate for the circularisation time of the system. Due to the low mass ratio $q=M_{2} / M_{1}$, we find a circularisation time of the order of the Hubble time even for this close binary system.

\section{MACHO 118.18793.469}

Two spectral components could be identified, each with $T_{\text {eff }}=$ $5400 \mathrm{~K}$ and a highly subsolar $([\mathrm{Fe} / \mathrm{H}]=-1.5)$ metallicity. For the light-curve and $R V$ fits with Nightfall we used the $R V$ amplitudes of the two stars to derive masses by the procedure described in the previous section. A reasonable fit to the $R V$ measurements folded to twice the period of DC can be achieved with sinusoidals (dashed curves in Fig. 1), i.e. assuming a circular orbit for the two components. However, an improved fit can be achieved by fitting the light-curve and radial velocities in Nightfall at the same time to an elliptical orbit (solid curves in Fig. 1). The best fit is achieved with a small eccentricity of $e=0.041$ and a periastron longitude of $\omega=89.94^{\circ}$. By varying the third light and the inclination, we construct the $\chi^{2}$-map shown in Fig. 3. As suggested by the spectral analysis of MACHO 118.18793.469, the lowest $\chi^{2}$-value is found for a third 

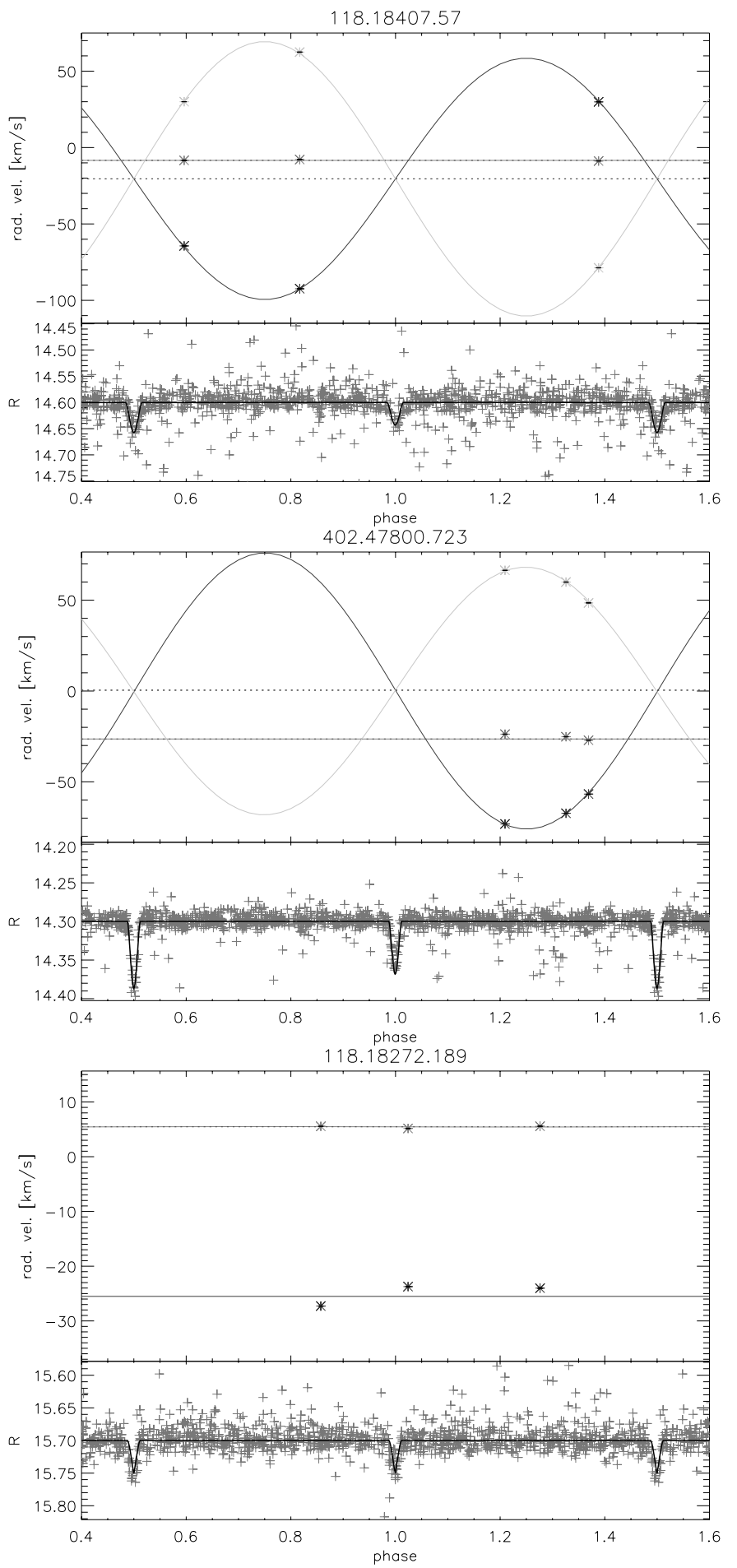

Fig. 2. Radial-velocity and light-curve fits for systems with circular orbits. Component $a$ is plotted in black, component $b$ in grey, and component $c$ in a lighter grey. The solutions shown are calculated assuming ZAMS stars. The error bars for the $R V$ measurements are of the size of the symbols.

light of zero. The inclination is $85.6^{\circ}$ for the ZAMS and $82.2^{\circ}$ for the TAMS. The low depths of the transit are therefore due to a grazing eclipse. This is also supported by the $\mathrm{V}$-shape of the best-fit model.

The best-fit light-curve model shows different transit depths. This is an indicator for two transits in one orbital period caused by two stars of slightly different size.

\section{MACHO 118.18407.57}

Three components are visible in the CCs of the three spectra, one of which shows $R V$ variations below $1 \mathrm{~km} \mathrm{~s}^{-1}$. Therefore, this is a third component, either in a wider orbit or physically unrelated to the other two. Component $a$ and $c$ show $R V$ changes of over $100 \mathrm{~km} \mathrm{~s}^{-1}$. They can be well-fitted with sinusoidals of twice the period given by DC, i.e. $4.7972 \mathrm{~d}$. If the photometric data are phased accordingly, we then see both transits where the transit depths are reduced due to third light of component $b$.

For the light-curve simulation we once more used the $R V$ amplitudes of $a$ and $c$ to get the masses and varied the inclination and third light coming from component $b$. The effective temperatures and radii of the components are interpolated from the Geneva evolution tracks assuming young stars on the ZAMS and older stars on the TAMS. The contribution of component $b$ meets the expectations from the spectral analyses $\left(T_{\text {eff }}=6200 \mathrm{~K}\right.$ for components $a$ and $c$ and $T_{\mathrm{eff}}=6600 \mathrm{~K}$ for component $b$, also see Fig. 3). The inclination of the system is $84^{\circ}$ assuming that the stars are on the ZAMS and $79.5^{\circ}$ for the TAMS. The system shows different transit depths, as MACHO 118.18793.469 does.

\section{MACHO 402.47800.723}

The second-brightest object in the sample shows three components in the spectra. Components $a$ and $c$ are best-fitted by a model with $T_{\text {eff }}=6400 \mathrm{~K}, b$ has $T_{\text {eff }}=5800 \mathrm{~K}$. As in the case of MACHO 118.18407.57, the masses are derived from the radial velocities. The $R V$ measurements of $a$ and $c$ are well-fitted assuming a circular orbit with twice the period of DC. The third component only shows small $R V$ variations and therefore seems to have a larger period than the other two. The fractional third light contribution for this component is $\sim 1 / 3$ and an inclination of the eclipsing system is $85.8^{\circ}$ assuming stars on the ZAMS and $82.3^{\circ}$ for the TAMS (see Fig. 3). We again see transit depth differences. Due to the high signal-to-noise ratio of the lightcurve, these are quite obvious and amount to $\Delta R=0.015$. This observation is also expected from the orbital semi-amplitude differences.

\section{MACHO 118.18272.189}

Each of the three FEROS spectra displays two components. The spectral analysis reveals that both components have a similar effective temperature $\left(T_{\text {eff }}=5800 \mathrm{~K}\right)$ and a subsolar metallicity of $[\mathrm{Fe} / \mathrm{H}]=-0.5$. The cross-correlation shows that component $b$ has a constant $R V$ of $\sim 5.46 \mathrm{~km} \mathrm{~s}^{-1}$ within the above mentioned statistical errors. Component $a$ shows $R V$ variations of $\sim 3.5 \mathrm{~km} \mathrm{~s}^{-1}$. Folding the $R V$ measurements to the orbital period given by $\mathrm{DC}$, one sees that the two components visible in the spectrum cannot be responsible for the transit in the lightcurve since one $R V$ point is very close to the transit. Here the two components should have almost the same $R V$. This is clearly not present in the data. The same is the case if we double the period (see Fig. 2). Thus, we exclude the scenario that the two visible components are responsible for the transit.

In another plausible scenario, we treat component $b$ as third light and assume that component $a$ is eclipsed by a low-mass object not visible in the spectra. However, if we fit a sinusoidal to the $R V$ points, in our solution the star would move away from the observer after the transit while it should do the opposite. We can therefore discard this scenario.

One could argue that the variations in $R V$ measured for component $a$ is caused only by systematic errors and that the eclipse 

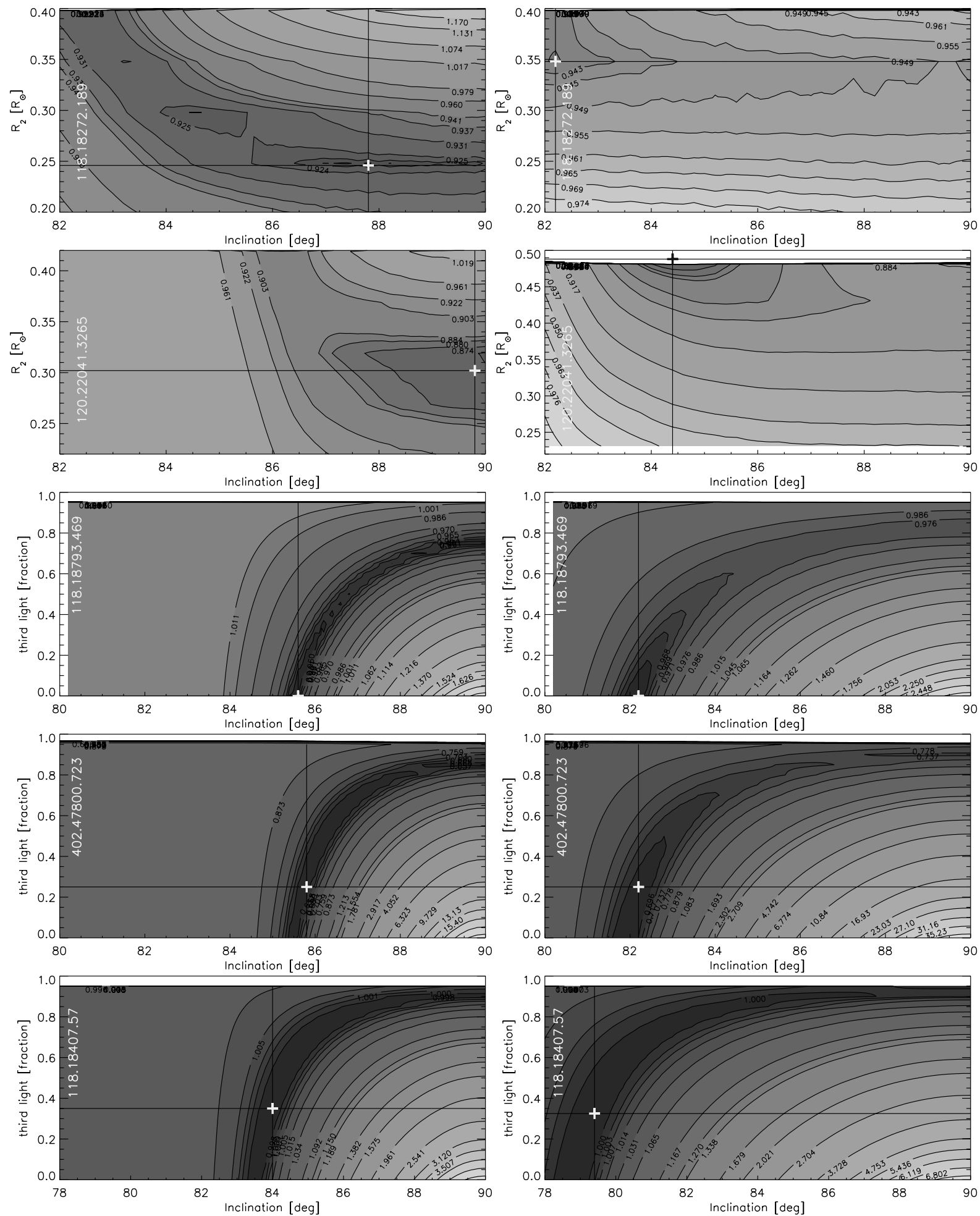

Fig. 3. $\chi^{2}$-contour plots for all analysed systems. In the left column we assume that the stars are on the zero-age main sequence and in the right column on the terminal-age main sequence. The bottom three plots show the $\chi^{2}$-contours for third light and inclination as fitted parameters. For the top two the radius of the eclipsing component and the inclination have been varied. The crosses mark best-fit values.

visible in the light-curve is caused by a planet orbiting $a$ without causing any noticeable $R V$ changes. We fitted this scenario taking the light from component $b$ into account and found a radius of $R_{2}=0.25 R_{\odot}$ assuming that $a$ is on the ZAMS and $R_{2}=0.35 R_{\odot}$ for $a$ being on the TAMS (see Fig. 3). These values, however, seem unrealistically high for planets and we can reject the 3-body scenario.
Finally, one scenario that can explain both the transit lightcurve and the measured $R V \mathrm{~s}$ is a four-body system consisting of the two $G$ stars which are visible in the spectra and two M-dwarfs invisible in the spectra. Here the two faint components orbit each other in twice the period from DC and eclipse each other twice. We assume an inclination of $90^{\circ}$ and two low-mass stars of equal size. The effective temperature of the 
eclipsing bodies was derived from the transit depth of the MACHO $R$-band light-curve using blackbody fluxes for all four components. The transit depth is reduced by the light of components $a$ and $b$. The $R V$ variations of component $a$ can in this scenario be explained by the reflex motion of $a$ to the orbit of the binary M-star system with a much larger period. We therefore do not observe a correlation between the transits and the $R V$. This scenario is underlined by the fact that the two $R V$ measurements in Fig. 2 at periods of $\sim 1.0$ and $\sim 1.3$, which have approximately the same $R V \mathrm{~s}$, are from two spectra taken only one day apart, while the third $R V$ value comes from a spectrum 26 days later. Component $b$ would be in a very wide orbit or physically unrelated to the other three stars.

\section{Summary}

For none of the five analysed MACHO-candidates could a planetary- or brown dwarf-companion be identified. We therefore confirm the speculation of DC that due to the depths of the transits in the photometric data the objects must be lowmass stars rather than sub-stellar objects. From the five candidates, we found one grazing eclipse of two nearly identical G-stars (MACHO 118.18793.469), two blends of deep eclipses of G-stars with a significant third light contribution (MACHO 118.18407.57 and MACHO 402.47800.723), one binary star with a G-type primary and an M-dwarf secondary (MACHO 120.22041.3265), and one rather complicated, blended system with four stars, of which each two are nearly identical (G- and M-type). With this work we show that for deeptransit surveys for extrasolar planets, follow-up observations to weed out false positives are efficiently possible with moderate effort.

To conclude, our results once again underline the need for spectroscopic follow-up of transit planet candidates as already shown by Bouchy et al. (2005) and Pont et al. (2005) for the OGLE survey and Torres et al. (2004) in the case of a blend scenario.

Acknowledgements. We would like to thank the referee for very useful comments.

S.D.H. gratefully acknowledges the support of the German-Israeli Foundation for Scientific Research and Development grant I-788-108.7/2003.

A.R. has received research funding from the European Commission's Sixth Framework Programme as an Outgoing International Fellow (MOIF-CT-2004002544).
This paper utilizes public domain data obtained by the MACHO Project, jointly funded by the US Department of Energy through the University of California, Lawrence Livermore National Laboratory under contract No. W-7405-Eng-48, by the National Science Foundation through the Center for Particle Astrophysics of the University of California under cooperative agreement AST-8809616, and by the Mount Stromlo and Siding Spring Observatory, part of the Australian National University.

\section{References}

Alcock, C., Axelrod, T. S., Bennett, D. P., et al. 1992, in Robotic Telescopes in the 1990s, ed. A. V. Filippenko, ASP Conf. Ser., 34, 193

Bakos, G. A., Noyes, R. W., Kovacs, G., et al. 2007, ApJ, 656, 552

Beaulieu, J.-P., Bennett, D. P., Fouqué, P., et al. 2006, Nature, 439, 437

Biller, B. A., Kasper, M., Close, L. M., Brandner, W., \& Kellner, S. 2006, ApJ, 641, L141

Bond, I. A., Udalski, A., Jaroszyński, M., et al. 2004, ApJ, 606, L155

Bouchy, F., Pont, F., Melo, C., et al. 2005, A\&A, 431, 1105

Charbonneau, D., Allen, L. E., Megeath, S. T., et al. 2005, ApJ, 626, 523

Charbonneau, D., Brown, T. M., Noyes, R. W., \& Gilliland, R. L. 2002, ApJ, 568,377

Chauvin, G., Lagrange, A.-M., Dumas, C., et al. 2004, A\&A, 425, L29

Chauvin, G., Lagrange, A.-M., Dumas, C., et al. 2005a, A\&A, 438, L25

Chauvin, G., Lagrange, A.-M., Zuckerman, B., et al. 2005b, A\&A, 438, L29

Collier Cameron, A., Bouchy, F., Hebrard, G., et al. 2007, MNRAS, 375, 951

Deming, D., Seager, S., Richardson, L. J., \& Harrington, J. 2005, Nature, 434, 740

Drake, A. J. \& Cook, K. H. 2004, ApJ, 604, 379

Gaudi, B. S., \& Winn, J. N. 2007, ApJ, 655

Gould, A., Udalski, A., An, D., et al. 2006, ApJ, 644, L37

Gray, D. F. 2005, The Observation and Analysis of Stellar Photospheres (Cambridge, UK: Cambridge University Press)

Hauschildt, P. H., Allard, F., \& Baron, E. 1999, ApJ, 512, 377

Marcy, G. W., Butler, R. P., \& Vogt, S. S. 2000, ApJ, 536, L43

Mayor, M. \& Queloz, D. 1995, Nature, 378, 355

McCullough, P. R., Stys, J. E., Valenti, J. A., et al. 2006, ApJ, 648, 1228

Neuhäuser, R., Guenther, E. W., Wuchterl, G., et al. 2005, A\&A, 435, L13

O’Donovan, F. T., Charbonneau, D., Mandushev, G., et al. 2006, ApJ, 651, L61

Pont, F., Bouchy, F., Melo, C., et al. 2005, A\&A, 438, 1123

Schaerer, D., Meynet, G., Maeder, A., \& Schaller, G. 1993, A\&AS, 98, 523

Schneider, J. 2002, Eur. Rev., 10, 185

Torres, G., Konacki, M., Sasselov, D. D., \& Jha, S. 2004, ApJ, 614, 979

Udalski, A., Jaroszyński, M., Paczyński, B., et al. 2005, ApJ, 628, L109

Vidal-Madjar, A., Désert, J.-M., Lecavelier des Etangs, A., et al. 2004, ApJ, 604, L69

Winn, J. N., Johnson, J. A., Marcy, G. W., et al. 2006, ApJ, 653, L69

Wolf, S., Laughlin, G., Henry, G., F. D., et al. 2007, ApJ, accepted

Wolszczan, A., \& Frail, D. A. 1992, Nature, 355, 145

Zahn, J.-P. 1977, A\&A, 57, 383 